\title{
An Analytical and Experimental Comparison of the Flow Field of an Advanced Swept Turboprop
}

Harvey E. Neumann, L. J. Bober, and J. S. Serafini Lewis Research Center

Cleveland, Ohio

and

Li-Ko Chang

Purdue University

West Lafayette, Indiana

Prepared for the

Twenty-First Aerospace Sciences Conference sponsored by the American Institute of Aeronautics and Astronautics

Reno, Nevada, January 10-13, 1983

\section{N/SA}




\author{
Harvey E. Neumann, Lawrence J. Bober, and John S. Serafini \\ National Aeronautics and Space Administration \\ Lewis Research Center \\ Cleveland, Ohio \\ and \\ Li-Ko Chang \\ Purdue University \\ West Lafayette, Indiana
}

\begin{abstract}
An argon ion laser velocimeter (LV) with four beams has been used to measure the detailed flowfield of an advanced eight blade propeller with $45^{\circ}$ of tip sweep in the Lewis $8 \times 6$ Foot Supersonic Wind Tunnel. The propeller was tested at a Mach number of 0.8. The propeller operated at an advance ratio of 3.06 and a blade angle at the three-fourths radius of $60.5^{\circ}$. The test configuration was also analyzed using a curved lifting line analys is. A comparison of analys is results with LV data indicated blade blockage occurred near the nacelle. This resulted in a deceleration ahead of the blade and unsteady flow downstream. The LV data also indicated the presence of a shock wave emanating from the suction surface at a radius ratio of 0.79 . The curved lifting line analysis qualitatively predicted the SR-3 propeller flow field. The comparison showed some differences between prediction and LV measurements. These differences can be attributed to phenomena not included in the analys is such as viscous blade wakes, tip vortex rollup, blade blockage, and nacelle effects. The comparison has indicated underpredictions in the magnitude of the radial velocity jump across the blade. These differences lead to differences in blade loading and wake location.
\end{abstract}

\section{Symbols}

$C_{p} \quad$ power coefficient $=P / \rho_{0} N^{3} J^{5}$

$\mathrm{D}$ blade tip diameter, cm (in.)

$\mathrm{J}$ advance ratio, $\mathrm{V}_{0} / \mathrm{nD}$

$M \quad$ Mach number

N rotational speed, revolutions per second

$\mathrm{P} \quad$ power, $\mathrm{kW}(\mathrm{ft}-\mathrm{ib} / \mathrm{sec})$

$r$ radius, cm (in.)

$\mathrm{R}$ blade tip radius, $\mathrm{cm}$ (in.)

$v_{0} \quad$ free-stream velocity, $\mathrm{m} / \mathrm{sec}(\mathrm{ft} / \mathrm{sec})$

$v_{t} \quad t i p$ rotational velocity, $\mathrm{m} / \mathrm{sec}(\mathrm{ft} / \mathrm{sec})$

$x$ axiar distance from nose of spinner, $\mathrm{cm}$ (in.)

$\theta$ ratio of total temperature to standard sea-level temperature of $518.7^{\circ} \mathrm{R}$

oo free-stream density, $\mathrm{kg} / \mathrm{m}^{3}$ (slugs $/ \mathrm{ft}^{3}$ )

\section{Introduction}

Increased concern over fuel costs and the national need to conserve energy has led to a renewed interest in turboprop propulsion for high speed subsonic aircraft. Numerous studies by industry and NASA (which are summarized in Ref. 1) have shown that there is a potential 15 to 25 percent trip fuel savings with advanced turboprop powered aircraft compared to equivalent technology turbofan powered systems for aircraft that cruise at Mach 0.8 . To achieve this fuel savings advantage, the propeller on this advanced turboprop would have to incorporate a number of unique design features that could enhance propeller performance and lower source noise at this high speed (2). Some of these design features are: large number of thin highly swept blades, high power loading (power/diameter squared), area-ruled spinner, and integrated propeller and nacelle. These design features lead to complex flow fields which are not amenable to conventional design analys is techniques. As new aerodynamic analyses are developed as part of NASA's Propeller Research Program, experimental data is necessary to evaluate and verify these new techniques.

This paper will present experimental data for one of NASA'S more promising advanced propeller designs, the SR-3. A recent study (3) has shown that this propeller has both good noise characteristics and high aerodynamic efficiencies. The SR-3 propeller has been studied using a laser velocjmeter (LV) to determine the flow

field(4). This paper is a continuation of that study and presents both an analys is of the flow field and a comparison with the predicted results from a curved lifting $l$ ine analysis.

\section{Experimental Apparatus and Procedure}

\section{SR-3 Propeller}

Figure 1 shows the SR-3 propeller, spinner and nacelle mounted on the Lewis 1000-hp Propeller Test Rig and installed in the test section of the NASA Lewis $8 \times 6$ Foot Supersonic Wind Tunnel. The propeller has eight blades with $45^{\circ}$ of sweep at the tip. The SR-3 has a $62.2 \mathrm{~cm}(24.5 \mathrm{in}$.) tip diameter. All experimental data were obtained at a tunnel Mach number of 0.8 and at the design advance ratio of 3.06 . The corrected tip rotational speed $\left(V_{t} / \sqrt{\theta}\right)$ was $280 \mathrm{~m} / \mathrm{sec}(917 \mathrm{ft} / \mathrm{sec})$ and helical tip Mach number was 1.15. The propeller was operated at a power coefficient of 1.8 , which was obtained at a blade angle setting at the three-fourths radius of $60.5^{\circ}$. The SR-3 geometry and its wind tunnel performance are given in Ref. 5 .

\section{Laser Velocimeter System}

The major components of the laser velocimeter system are shown in Fig. 2 and are described in Refs. 4 and 6. A 15-watt argon ion laser is operated at about $1 / 3$ power. The system uses a four beam on-axis backscatter optic system. The measuring volume is moved in two directions by traversing the entire laser system and is moved in the third direction using the zoom lens assembly. The movement of the measuring volume is remotely 
computer controlled. The flow with in the tunnel is artificially seeded with dioctyl phthalate $(0.0 . P$.$) . The four beam laser velocimeter is cap-$ able of measuring two velocity components simultaneously. The four beams were set up such that the planes defined by the two beams of each color were essentially orthogonal to each other and at nominally $45^{\circ}$ to the horizontal plane of the wind tunnel. The axial and tangential components of velocity were obtained by making a measurement in the horizontal plane which passes through the propeller rotational axis. The axial and radial components of velocity were similarly obtained by making measurements in the vertical plane which passes through the rotational axis.

\section{Analys is Method}

The analysis method used in the comparison is the curved lifting line analys is presented in Refs. 7 and 8 . This analys is has been extended to include the effects of blade drag, camber and radially varying inflow into the propeller. The radial variation of inflow velocity is determined from an jnviscid axisymmetric transonic analysis $(9,10)$, of the flow around the nacelle with no blades present. The curved lifting line analysis does not otherwise consider the nacelle. A study of the curved lifting line analys is for advanced propeller designs(19), has analytically investigated the sensitivity to various assumptions in the analysis. In the analys is, each propeller blade is represented by a bound vortex with radially varying strength over the blade span. This radially varying strength causes vorticity to be shed from the blade and transported downstream forming a helical vortex sheet. In practice, the bound vortex is divided into a finite number of elements, each having constant vorticity with a shed vortex filament originating from each element end point. Each shed vortex is assumed to be a helix with constant pitch. Since the geometry of the wake is completely specified, there is no provision in the analysis for the outer portion of the wake near the tip to roll up into the tip vortex. A tip vortex, without rollup, is associated with the edge of the vortex sheet and is included in the analysis. The strengths of the bound vortex elements are determined by requiring that the flow be tangent to the blade at certain control points. The bound vortex elements are placed along the one quarter chord line and an equal number of control points are placed along the three quarter chord line. The influence of the bound and shed vortices at the control points are determined using the Biot-Savart relationship resulting in a series of simultaneous linear equations which are solved for the strengths of the bound vortex elements. This then defines the strength of the shed vortex filaments. The induced flow at the curved lifting line is then calculated from the known vortex strengths and is added to the propeller inflow velocity and rotational velocity to determine the total velocity. Velocities at arbitrary points in the flow field are calculated using the Biot-Savart relationship along with the known strengths of the bound and shed vortex filaments.

\section{Resuits and Discussion}

The experimental results presented are part of an extensive set of data obtained for the flow field of the SR-3 propeller at its design point during transonic operations at Mach 0.8. Additional results are given in Ref. 4. The propeller was operating at an advance ratio of 3.06 , a power coefficient of 1.8 and with a blade angle at the three-fourths radius of $60.5^{\circ}$. The experimental results are compared to predictions from the curved lifting line analysis. The velocity data is presented in terms of a cylindrical coordinate system centered along the propeller model centerline with the positive axial direction in the downstream direction (Fig. 3). The origin of the system is at the propeller spinner nose.

Measurements of flow field velocities upstream and downstream of the propeller were made at three axial locations and one radial location, as shown in Fig. 3. A comparison of $L V$ measurements of entrance velocity of the SR-3 propelier with predictions from an inviscjg axjsymmetric transonic analys is without blades $(9,10)$, is given in Fig. 4. The results are presented in terms of Mach number distributions at two upstream measuring stations. At the upstream measuring station $(x / r$ $=0.09$ ), the agreement between theory and exper $\mathrm{i}-$ ment is excellent. The comparison at a $x / r=0.33$ station shows differences near the nacelle. The analysis, not including the effects due to the blades, shows a higher Mach number than the data. These results indicate that the flow near the nacelle is retarded due to the thickness of the blades (b lockage effects). The compar ison shows good agreement near the mid-span and tip of the blades. The results from this inviscid transonic analysis are used in the curved lifting line analys is to provide an estimate of the nonuniform entrance flow due to nacelle effects. Thus, it may be necessary to include blade blockage when determining propelier inflow velocities.

The flow behind the propeller at an $x / R=0.89$ has been measured with the LV system and compared with predictions from the curved lifting line analysis. Limited numerical comparisons of the velocity components are given in Fig. 5. Comparisons of the three velocity components are presented at radii just outside the propeller tip and near the mid-span. Data is also given for flow near the nacelle at a radius ratio of 0.35 . Data at this radial station is oownstream of where blade blockage effects were noted in the entrance flow. Close examination of the axial and radial velocities have shown no blade-to-blade correlation or repeatability near the nacelle. Large data scatter have also been noted. This implies that the flow is unsteady (in the relative velocity frame). The nacelle curvature and the flow blockage result in the relatively large radia) components shown in $\mathrm{Fig}$. 5 (b) at a radius ratio of 0.35 . A comparison of the LV data with the curved lifting line results has not been presented at this radial position because the unsteady flow rencers the comparison meaningless.

At radius ratios of 0.65 and 1.03 , the comparison shows generaliy good agreement in predicted and measured axial velocity, Fig. 5(a). The curved lifting line analys is does not include viscous wake and tip vortex roll-up effects. The viscous wake shown in the data $(a t, r / R=0.65)$ at a circumferential position of $10.5^{\circ}$ cannot therefore be predicted. The tip vortex is stronger in the analysis resuits than in the data because of the mathematical singularity present in the analy$\mathrm{sis}$ at the tip. It should, however, be noted that the jump in velocity across the blade is underpredicted and the analys is predicts the wake angular location to be at about $18^{\circ}($ at $r / R=0.65)$ 
rather than the measured $9^{\circ}$. The discrepancy in wake location is also in evidence in the radial and tangential velocities, Figs. 5(b) and (c). The general level of the radial and tangential velocities are also not well predicted by the curved lifting line analysis. These two components, however, are relatively small compared to the axial velocity ano therefore do not seriously impair the total velocity comparison.

A color computer graphic technique has been utilized to evaluate and compare the measurements and predictions of the highly three dimensional exit flow from the propeller. LV data from circumferential surveys at 17 radial positions from $r / R=0.59$ to 1.22 were used to generate the computer graphic presentations of Fig. 6 at the exit measuring station, $x / R=0.89$. Only data beyond the mid span was available for use in this graphical representation. At each radial position, the data over the complete circumference for the 8 blades was "folded" to a $45^{\circ}$ segment to provide data in a single "equivalent" blade passage. The circumferential data was averaged to provide 30 circumferential values with in this "equivalent" blade passage. The entire array of experimental data at 17 radial positions by 30 circumferential positions was interpolated to provide data at intermediate positions, color-coded and displayed on a color raster display. The results were photographed to provide the results shown in the figure. The analytical results were processed in an identical manner. Velocities were predicted for the same 17 by 30 array of spatial positions, computer interpolated and graphically displayed.

Figure 0 presents the velocity fields for each velocity component in both the absolute reference frame seen by a stationary observer and for the relative reference frame seen by an observer $r i d-$ ing the blades. The blade rotation in the figure is in the counterclockwise direction. The axial velocity in Fig. 6(a) clearly show the thick blade wakes and the tip vortex. The yellow region adjacent to the blade wakes is the nigh velocity from the suction surface. The maximum suction surface velocity occurs at about 0.9 of the tip radius. In making the comparison of the LV data with the results from the curved lifting line analysis, the viscous blade wakes must be ignored since they are not included in the analysis. Again, the comparison shows the stronger predicted tip vortex as was previously noted. The general character of the flow field as measured is strikingly similar to the flow field predicted by the curved lifting line analysis. Some flow disturbances are noted beyond the blade tip. These velocity fluctuations may present a potential noise source.

The radial velocity field is given in the lower part of Fig. 6(a). The analys is shows much smaller radial velocities near the nacelle than found in the data. This primarily results from the nacelle curvature effect not being included in the anaiysis. Near the tip, the analys is is qualitatively similar to the LV data. Both show higher radial velocities on the pressure side of the blade than on the suction side.

The tangential velocities are given in Fig. $6(b)$. The analytical and experimental results are again similar over most of the blade span if one ignores the blade wakes in the experimental data. The LV data shows an angular displacement between the blade wake and the tip vortex. This effect is not seen in the predicted results. The effect arises from the tip vortex rollup which is not included in the analysis.

The total velocity comparison given at the bottom of Fig. 6(b) is very similar to the axial velocity previously discussed. This is because the axial velocity is the dominant component of the three velocity components forming the total velocity.

The relative velocity is compared in Fig. 6(c). If the viscous wakes are ignored in the comparison, both experimental relative velocities presented are very similar to their respective analytical counterparts. In general, the curved lifting line analys is shows good qualitative agreement with the measured LV data.

The circumferential distribution of interblade axial velocity is shown in Fig. 7. The results shown are for an axial distance of $x / R=$ 0.73 and a radius ratio of $r / R=0.79$. The data for the eight blades has been "folded" so the data covers an angular displacement of $45^{\circ}$. This "folded" data is repeated twice in the figure. The data station is at the 70 percent chord location and is immediately upstream of the high suction surface velocity region shown in Fig. $6(a)$. The data shows a jump in velocity across the blades of about $30.5 \mathrm{~m} / \mathrm{sec}(100 \mathrm{ft} / \mathrm{sec})$. The curved lifting line analysis shows only about half the actual jump and is not as abrupt. In the analysis, the load on the olade is concentrated at the quarter chord line while, in actuality, the load is distributed along the chord of the blade. The mean level of the distributions and the qualitative shape are nevertheless considered good. The velocity data shown is immediately ahead of a shock emanating from the suction surface.

The shock wave and the complete flow field in between the blades at a radius ratio of $r / R=0.79$ is shown in Fig. 8. The same computer analys is technique previously described is used to present the LV data and the predicted results. LV data is presented in the top half of the figure and the curved lifting line analytical results in the lower half. Three velocities are presented. The resultant velocity is the vector sum of the axial and tangential velocities shown. The flow direction is from left to $r i g h t$ and the blade rotation from top to bottom. A short portion of the flow ahead of the blade is shown in addition to the flow with in the blades and slightly downstream of the blades. The data shown is for axial distances of $x / R=0.32$ to $x / R=0.94$. The blade extends from $x / R=0.53$ to $x / R=0.82$. The results have been "folded" to an included angular displacement of $45^{\circ}$. For presentation purposes the "folded" results have been repeated twice in the figure to show two blade passages.

The LV data shows a rapid acceleration on the suction side of the blade. The numerical results indicate a shock just downstream of the high velocity region. The data indicate that the shock, however, does not extend completely across the blade passage. The curved lifting line analys is does not include provision for shocks although the analys is does indicate correctly the rapid acceleration on the suction surface. The LV data indicates mild accelerations upstream of the blades. This effect also appears in the analys is however the velocity gradients appear to be larger in the analysis. The tangential component of velocity shows an abrupt jump in the axial direction both in the data and the analysis. The analysis predicts the jump slightly upstream of that meas- 
ured. The larger gradients in the analysis results near the blade are due to the concentration of the load at the quarter chord of the blade, whereas in reality the load is distributed along the chord. Predicted variations in tangential velocity near the blade are larger than those measured. The general character of the velocity field, however, show qualitative agreement between the curved lifting line predictions and the measured LV results.

\section{Conclusions}

The flow field of an advanced $45^{\circ}$ sweep, eight-bladed propeller has been measured with a laser velocimeter during transonic operation in the NASA Lewis $8 \times 6$ Foot Supersonic Wind Tunnel. The propeller was tested at a Mach number of 0.8 . The propeller operated at an advance ratio of 3.06 and a blade angle at the three-fourths radius of $00.5^{\circ}$. The test configuration was also analyzed using a curved lifting line analys is and a comparison of the results showed the following results.

1. The curved lifting line analys is qualitatively predicted the SR-3 propeller flow field. The comparison showed some differences between prediction and LV measurements. These differences can be attributed to phenomena not included in the analys is such as viscous blade wake losses, tip vortex rollup, blade blockage, and nacelle effects.

2. The LV data indicated a deceleration ahead of the blade and unsteady flow (in the relative reference frame) downstream near the nacelle. This was interpreted as resulting from blade b lockage.

3. The LV data indicated the presence of a weak shock emanating from the suction surface at a radius ratio of 0.79 . The data indicate that the shock does not extend completely across the blade passage.

4. LV data indicated that the flow approaching the propeller was steady; however, weak fluctuations in velocity were noted downstream of the propeller beyond the tip radius. These fluctuations may contribute to propeller noise.

5. The curved lifting line analys is includes effects of radially varying inflow to the propeller, but does not include effects arising from nacelle curvature. This leads to incorrect predictions of radial velocity near the nacelle. The comparison has also indicated that underpredictions in the magnitude of radial velocity jump across the blade occur. Differences in blade loading and wake location then result.

\section{References}

1. G. A. Mitchell and D. C. Mikkelson, "Summary and Recent Results From the NASA Advanced High-Speed Propeller Research Program, "NASA TM-82891, June 1982.

2. R. J. Jeracki and G. A. Mitchell, "Low and High Speed Propellers for General Aviation Performance Potential and Recent Wind Tunnel Test Results," SAE Paper 810601, April 1981.

3. R. J. Jeracki, D. C. Mikkelson, and B. J. Blaha, "Wind Tunnel Performance of Four Energy Efficient Propellers Designed for Mach 0.8 Cruise," SAE Paper 790573, April 1979.

4. J. S. Serafini, J. P. Sullivan, and H. E. Neumann, "Laser-Velocimeter Flow-Field Measurements of an Advanced Turboprop, "AIAA Paper 81-1568, July 1981 .

5. C. Rohrbach, F. B. Metzger, D. M. Black, and R. M. Ladden, "Evaluation of Wind Tunnel Performance Testing of an Advanced $45^{\circ}$ Swept Eight-Bladed Propeller at Mach Numbers From 0.45 to 0.85 ," NASA CR-3505, March 1982.

6. R. J. Freedman and J. P. Greissing, "Development and Utilization of a Laser Velocimeter System for a Large Transonic Wind Tunnel," NASA TM-82886, June 1982.

7. L. K. Chang, "The Theoretical Performance of High Efficiency Propellers," Ph.D. Thesis, Purdue University, December 1980.

8. J. P. Sullivan, "The Effects of Blade Sweep on Propeller Performance," AIAA Paper 77-716, June 1977.

9. J. D. Keller and J. C. South, Jr., "RAXBOD: A Fortran Program for Inviscid Transonic Flow Over Axisymmetric Bodies," NASA TM X-72831, February 1976 .

10. J. C. South, Jr, and A. Jameson, "Relaxation Solutions for Inviscid Axisymmetric Transonic Flow Over Blunt or Pointed Bodies," AIAA Computational Fluid Oynamics Conference ( $\mathrm{Palm}$ Springs, California, July 1973, pp. 8-17)

11. L. K. Bober and L. K. Chang, "Factors Influencing the Predicted Performance of Aovanced Propeller Designs, " AIAA Paper 81-1564, July 1981 . 


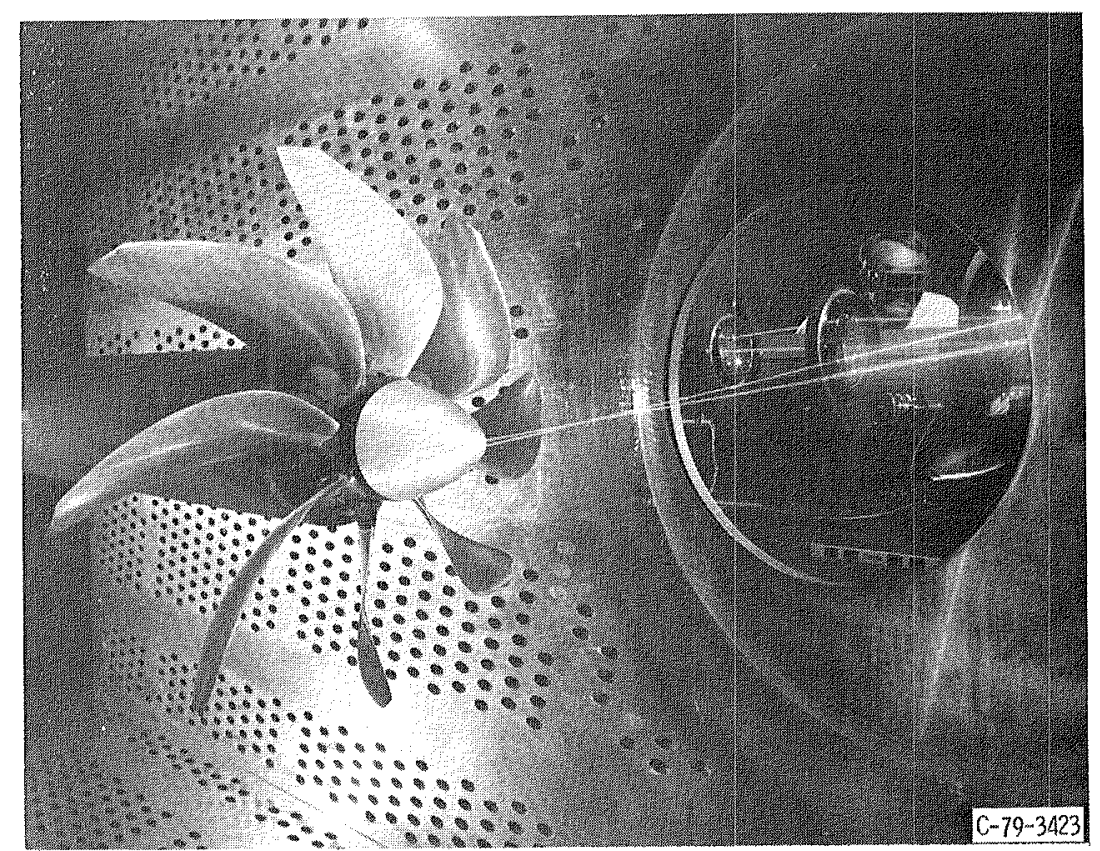

Figure 1. - SR-3 propeller installation in NASA Lewis 8- by 6-foot Supersonic Wind Tunnel.

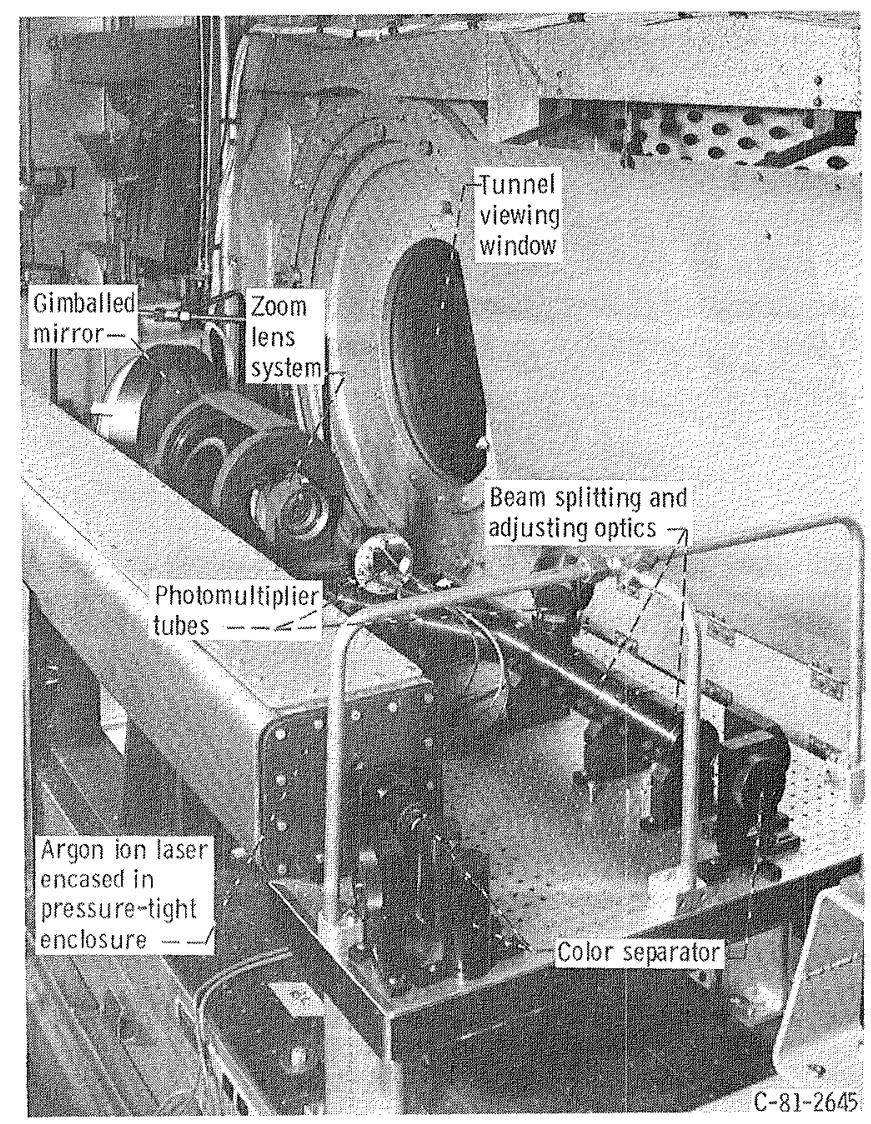

Figure 2. - Components of laser velocimeter system installed in NASA Lewis 8- by 6-foot Supersonic Wind Tunnel. 


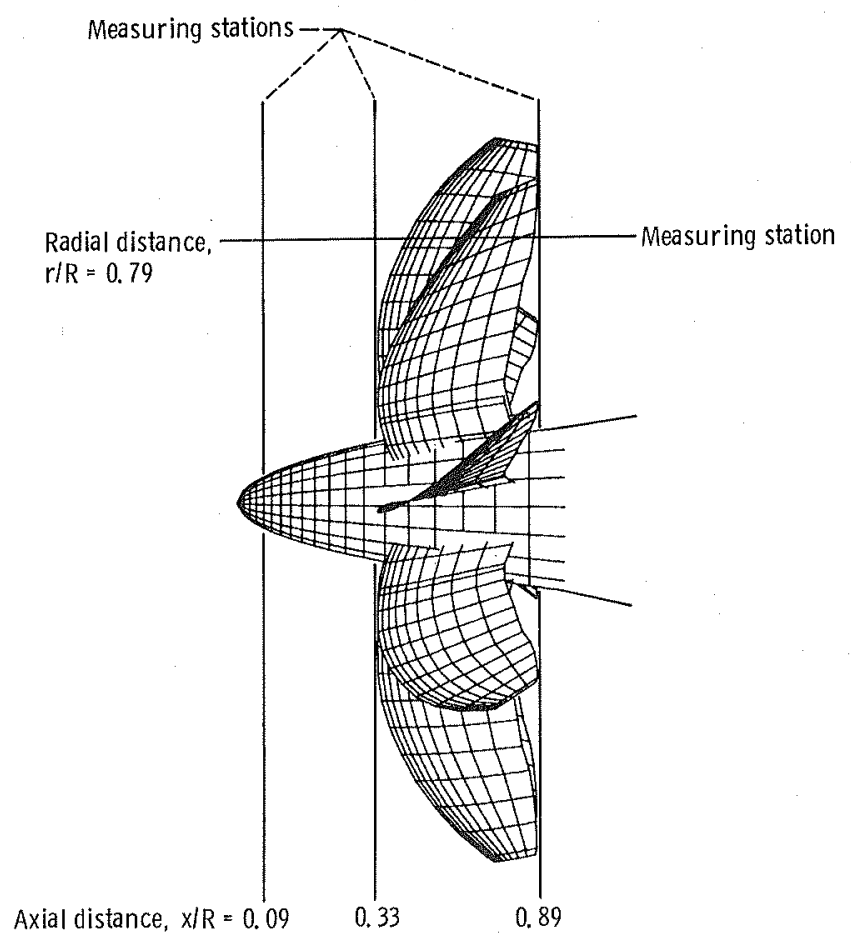

Figure 3. - Sketch of SR-3 propeller and nacelle showing LV measuring stations.

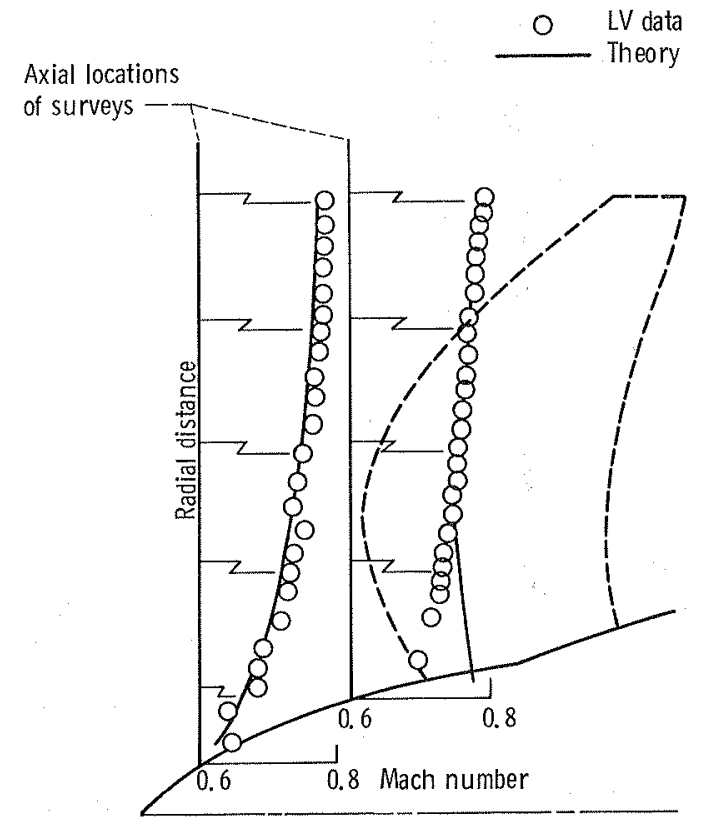

Figure 4. - Comparison of LV measurements of Mach number with an inviscid transonic analysis without blades for the SR-3 propeller. 

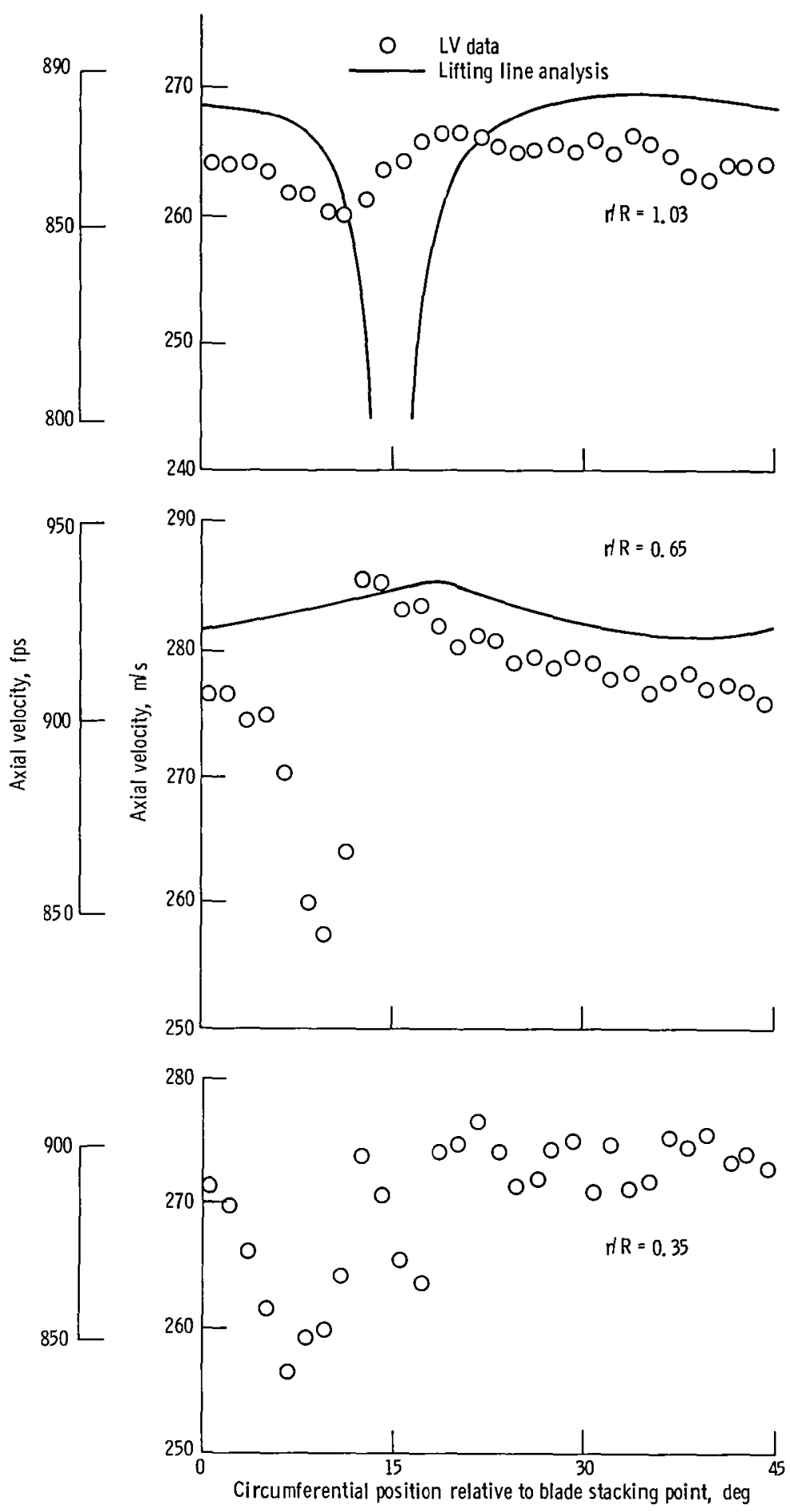
(a) Circumferential distribution of axial velocity.

Figure 5. - Comparison of LV measurements of velocity distributions at SR-3 propeller exit $(X / R=0.89)$ with lifting line analysis. 

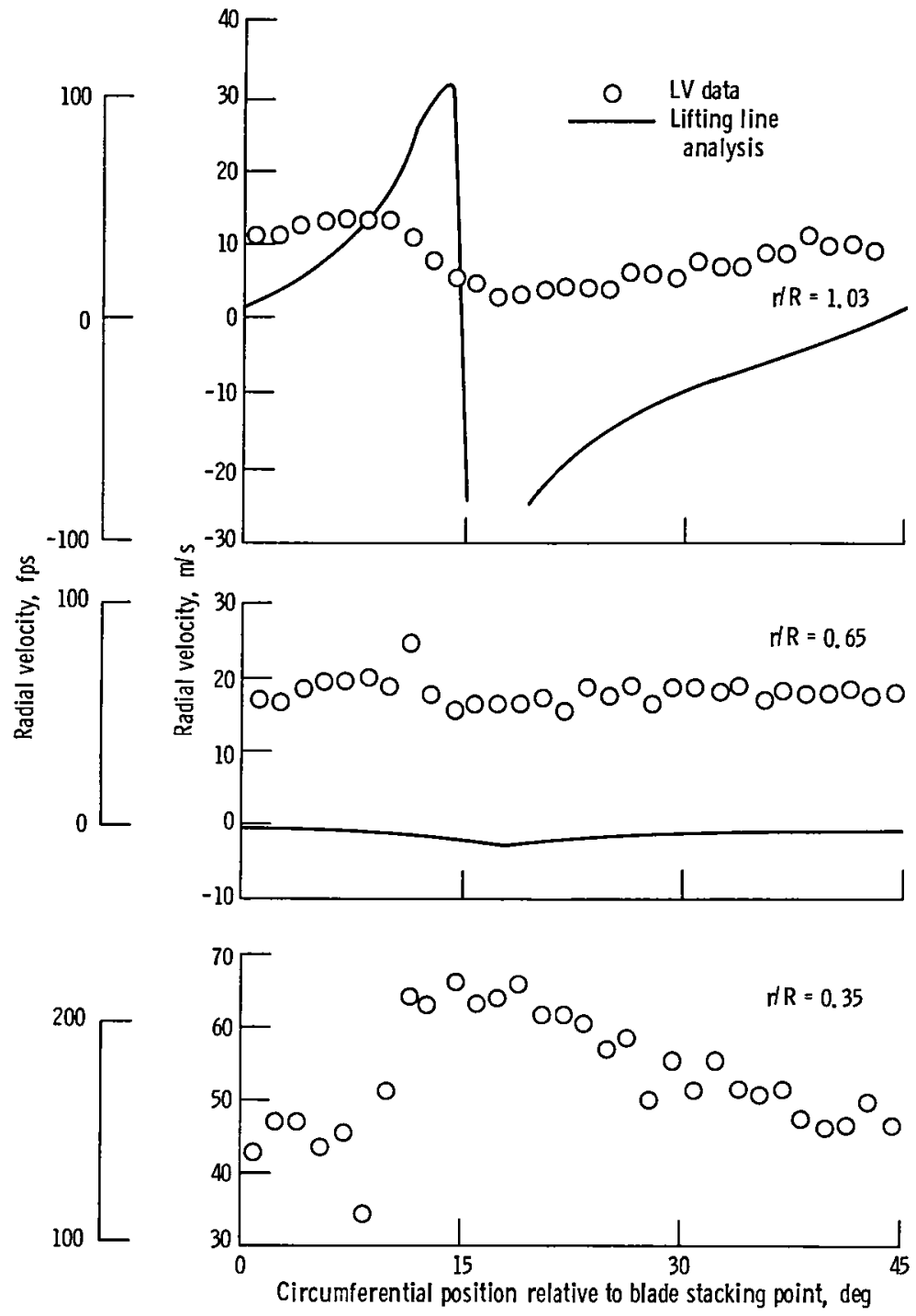
(b) Circumferential distribution of radial velocity.

Figure 5. - Continued. 


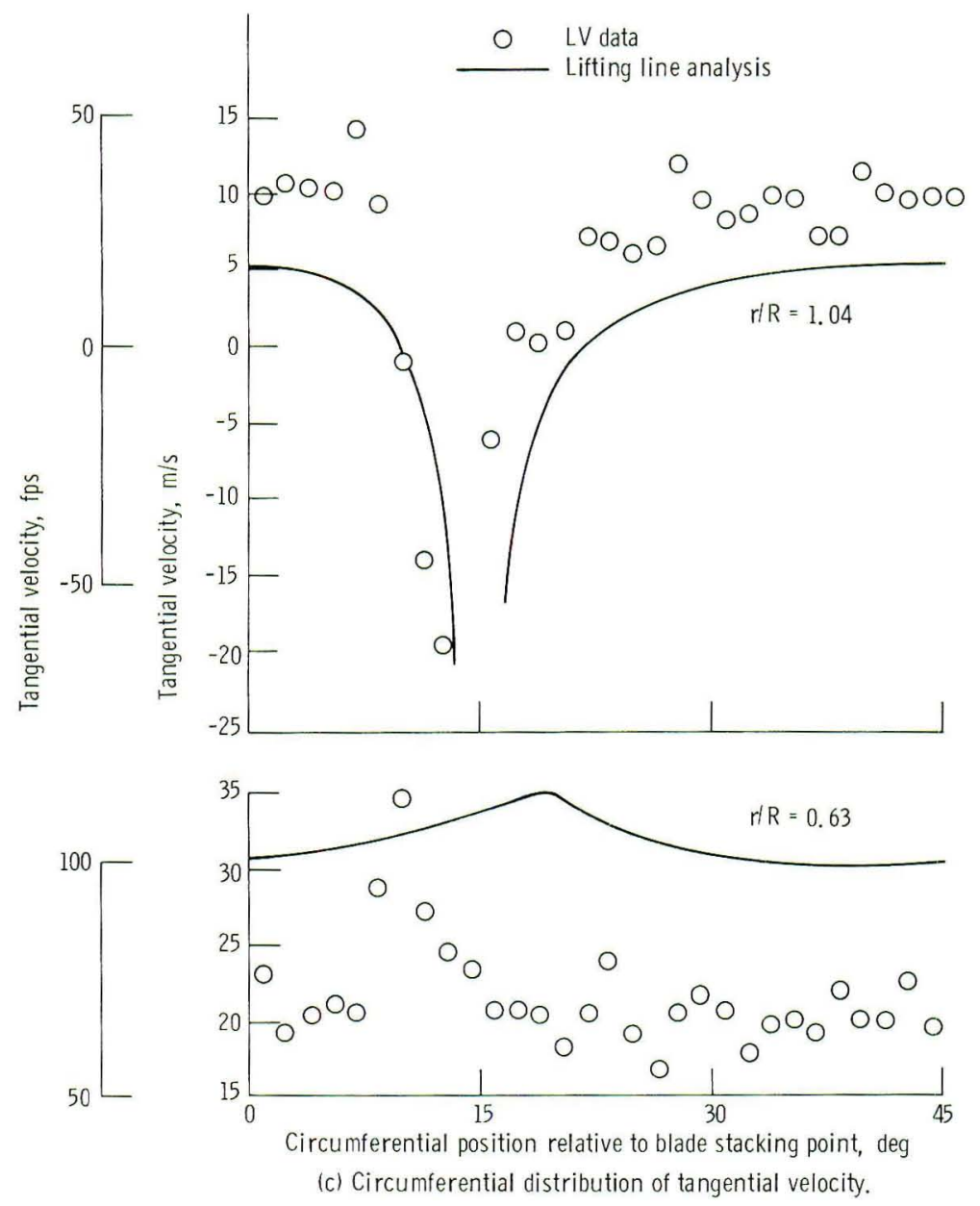

Figure 5. - Concluded. 


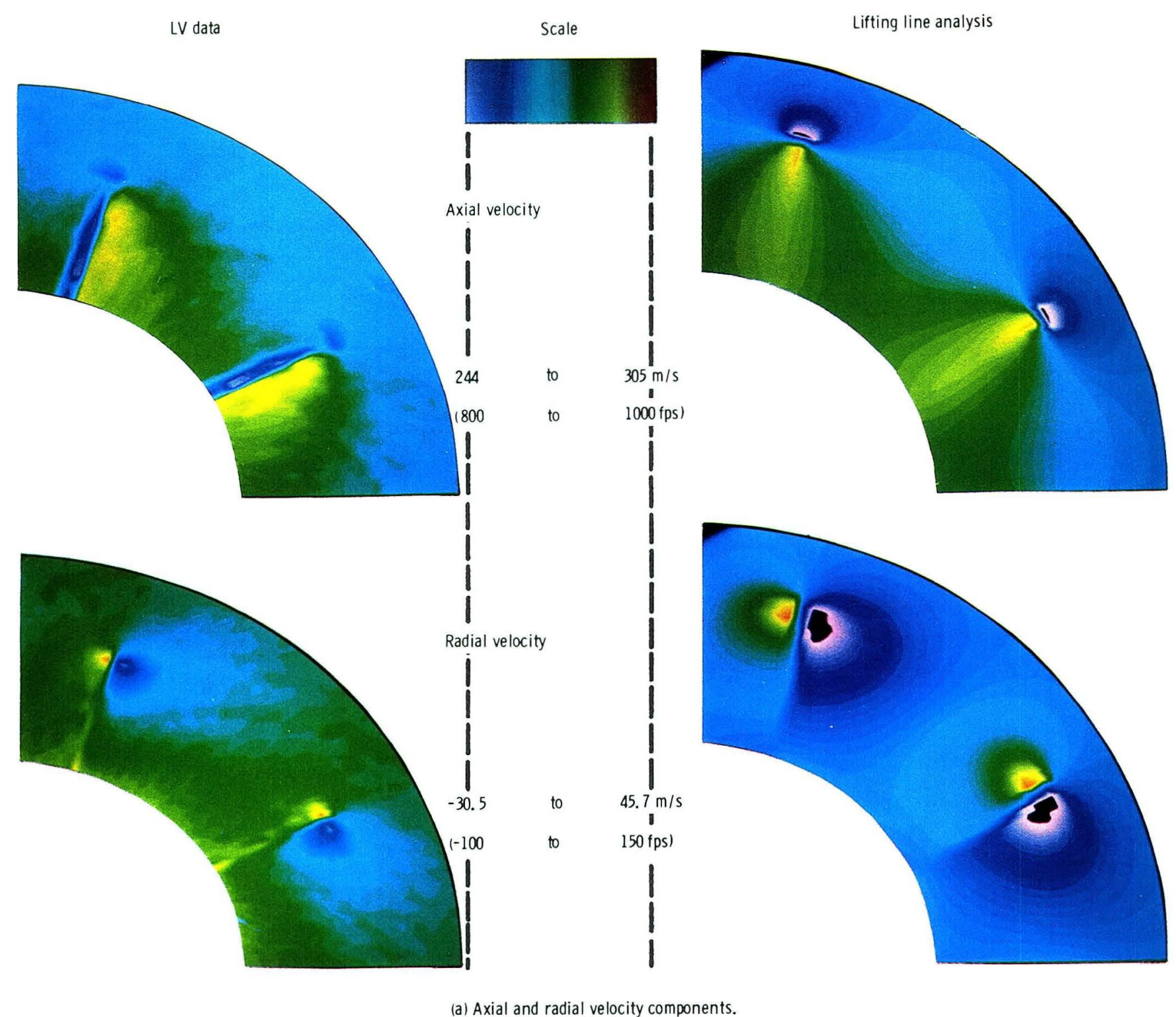

Figure 6. - Comparison of measured and predicted velocities at exit of SR-3 propeller. 


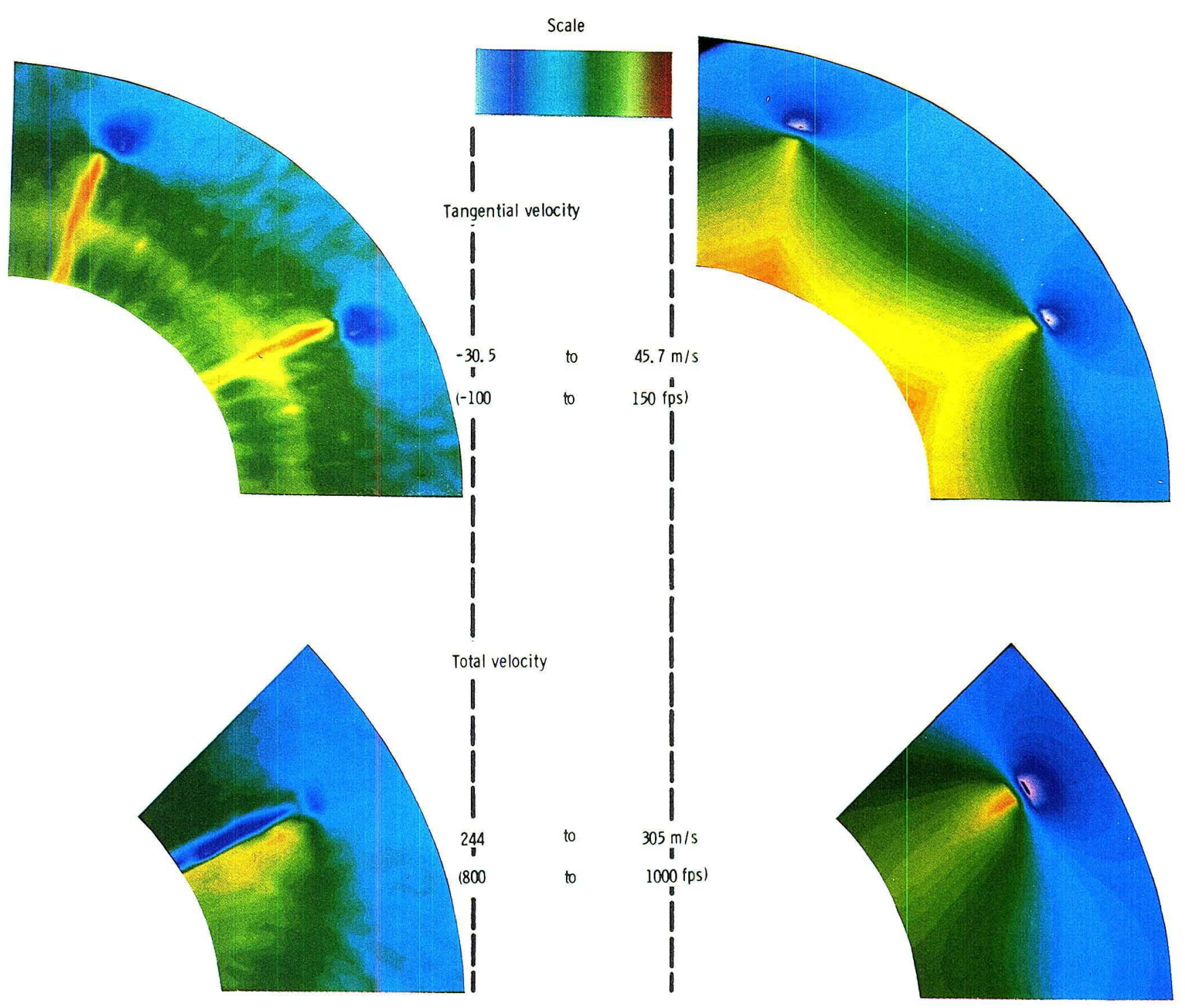

(b) Tangential and total velocities.

Figure 6. - Continued. 


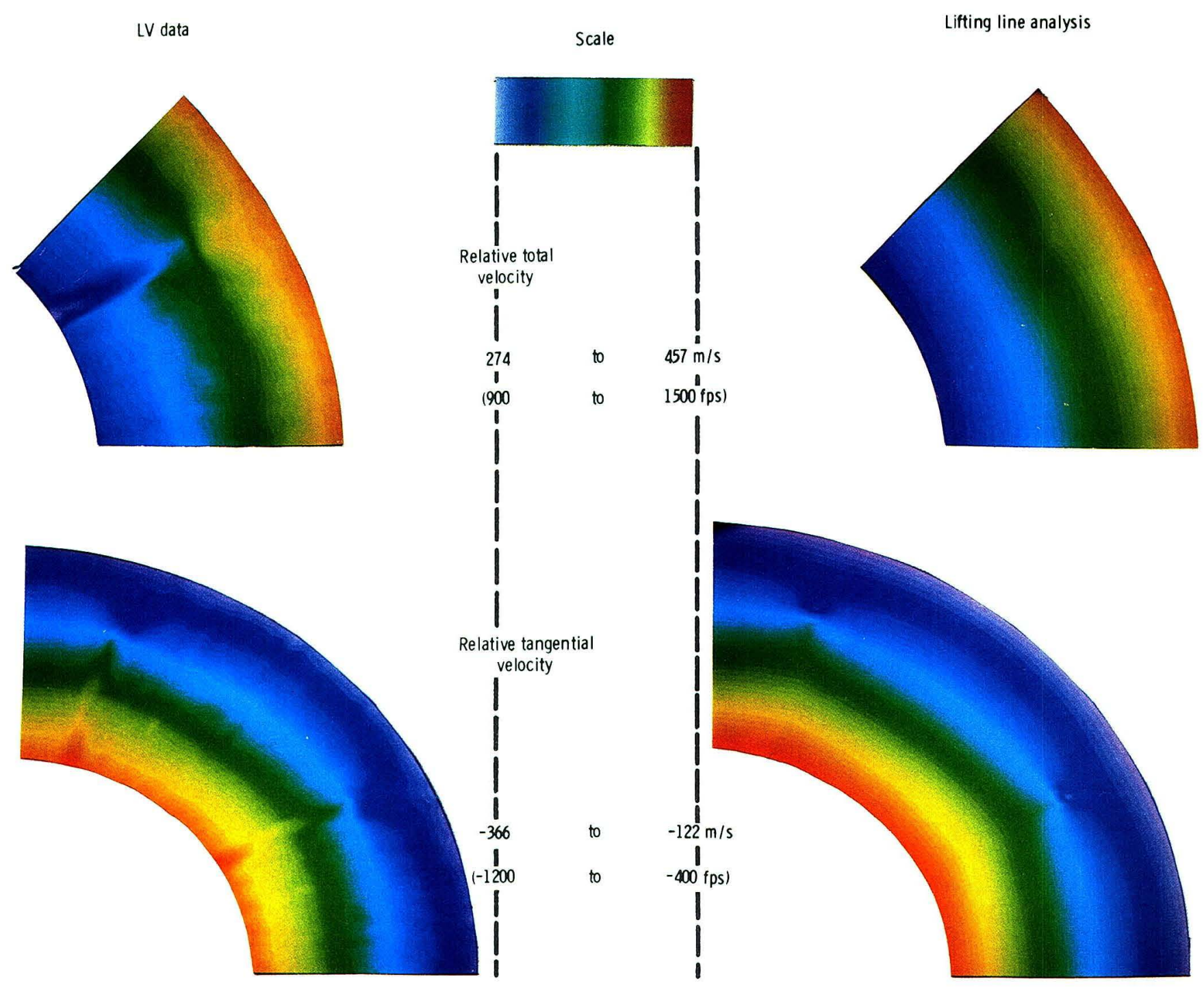

(c) Relative total and relative tangential velocities.

Figure 6. - Concluded. 


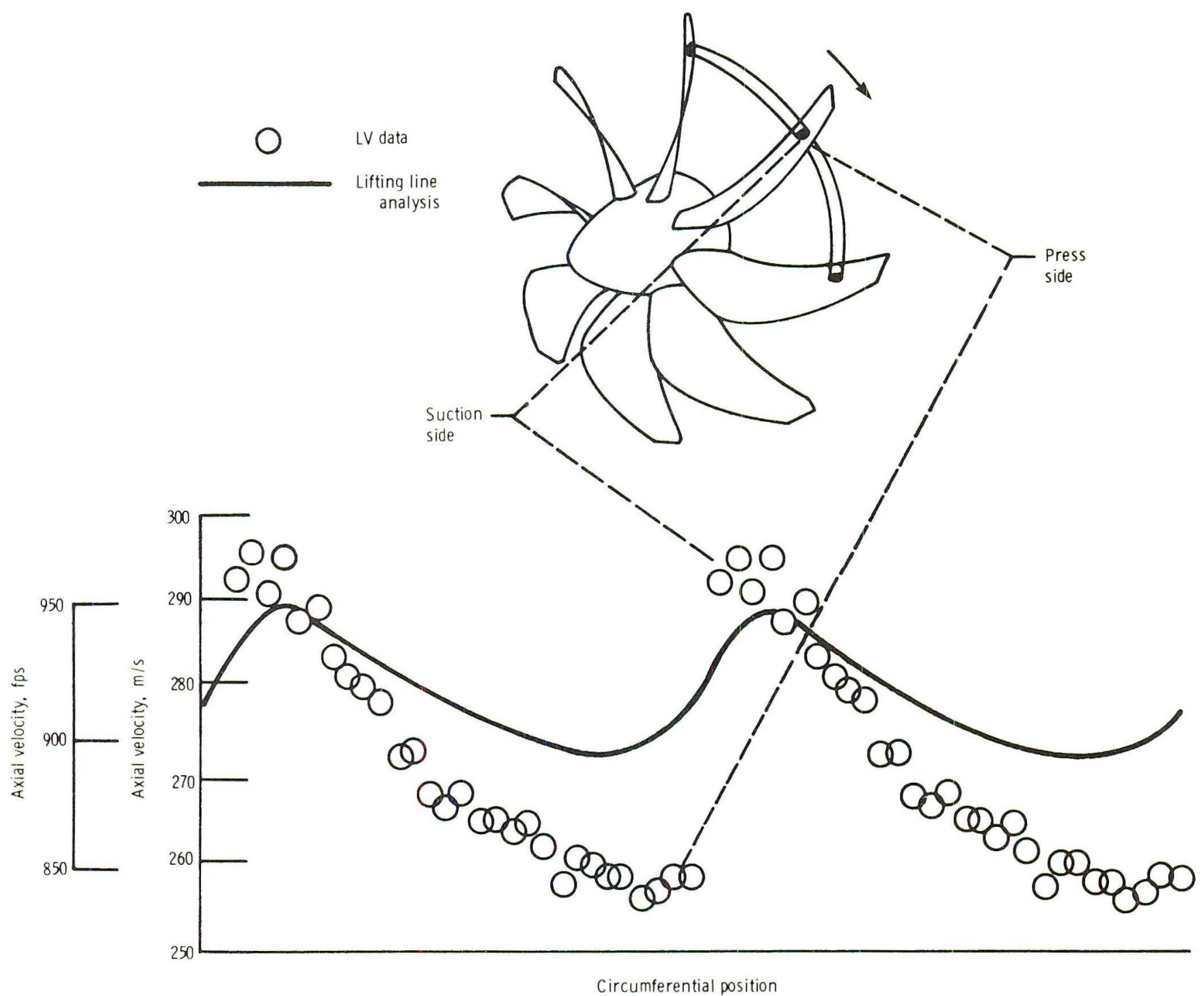

Figure 7. - Comparison of measured and predicted circumferential distribution of axial velocity with in SR-3 propeller blades: $r / R=0.79$; $X / R=0.73 . M=0.8 ; C_{p}=1.8 ; J=3.06$. 


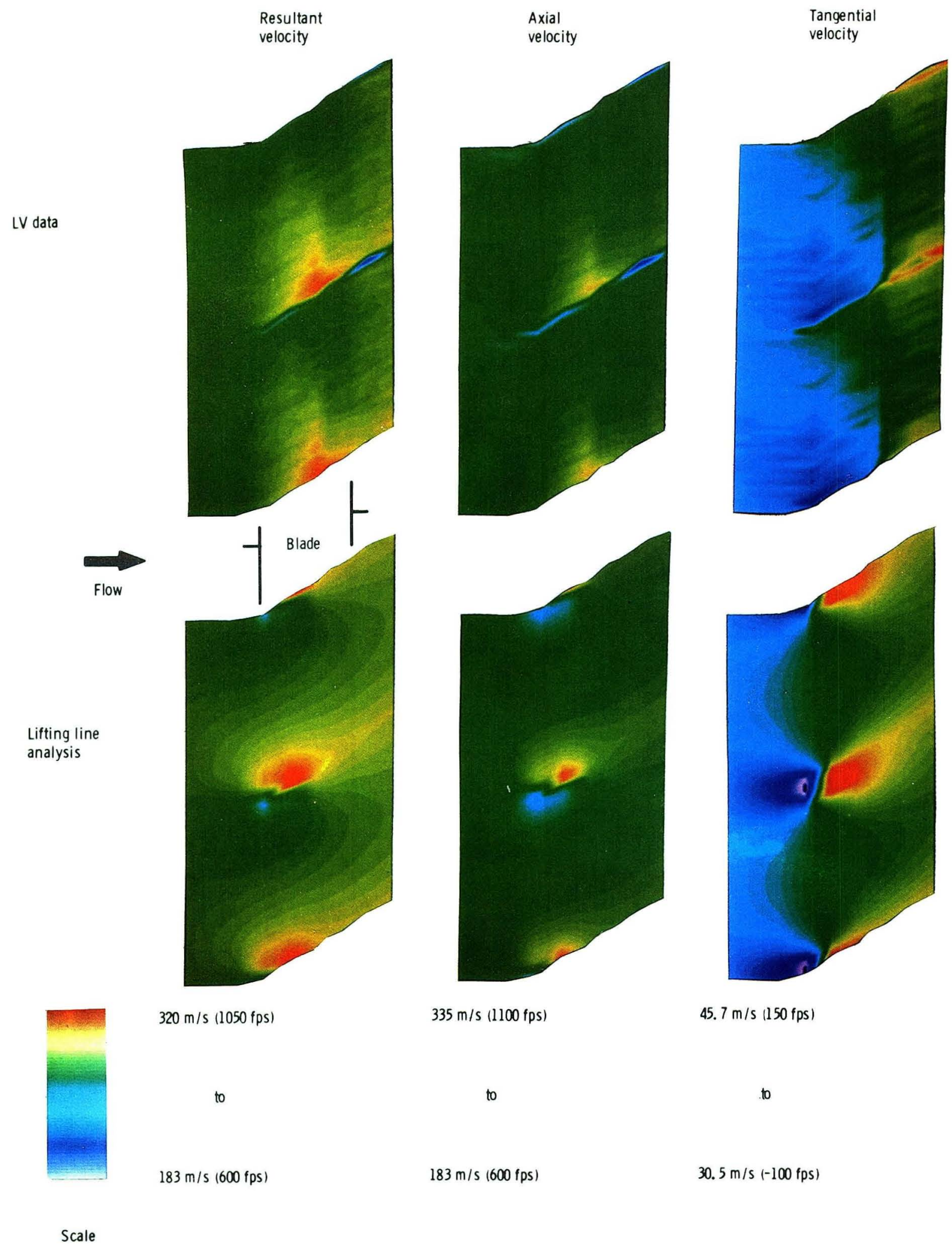

Figure 8. - Comparison of measured and predicted interblade velocities; $r / R=0.79$. 


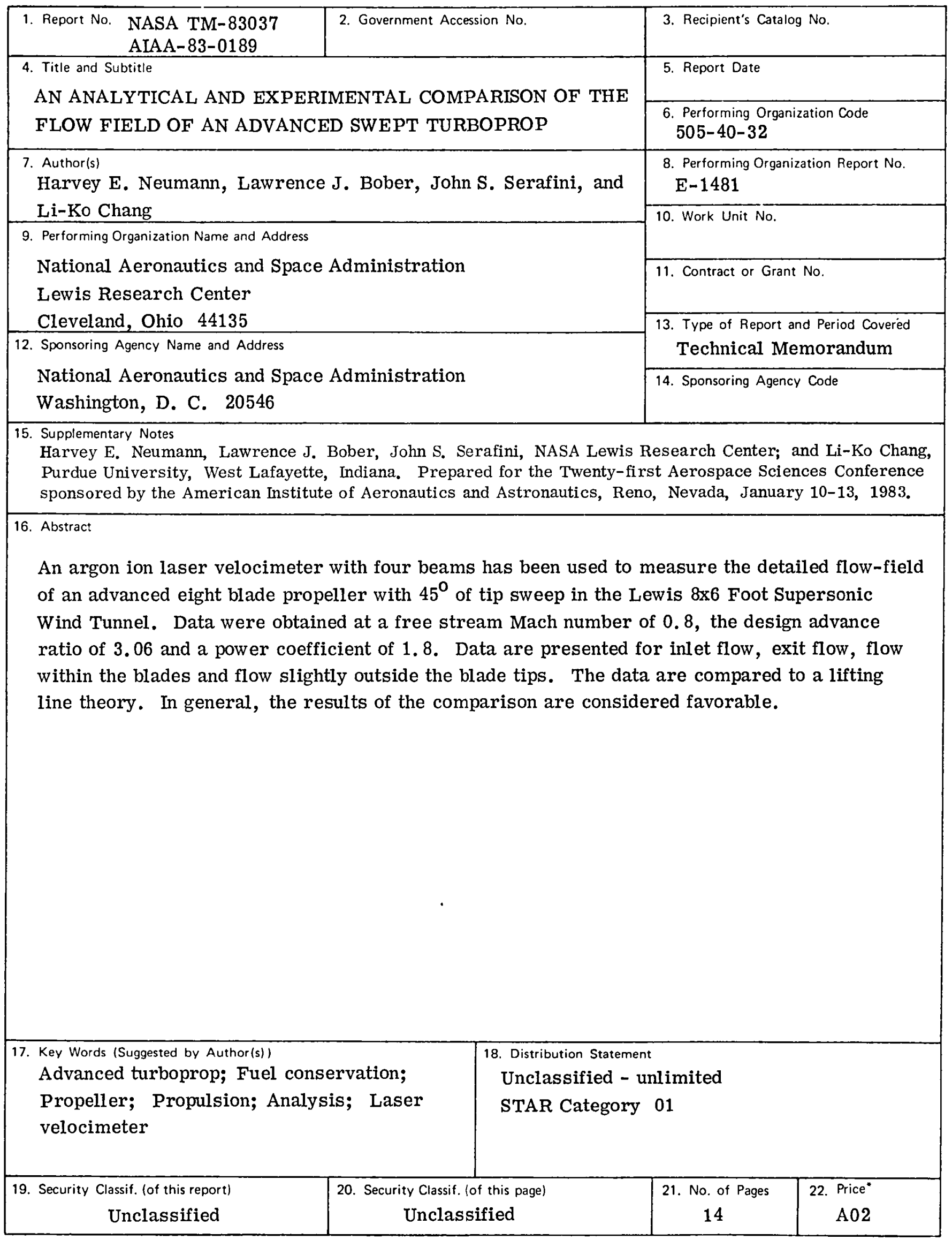

* For sale by the National Technical Information Service, Springfield, Virginia 22161 
End of Document 